\title{
CONTRIBUCIÓN AL ESTUDIO FAUNÍSTICO DEL BENTOS FLUVIAL DEL RÍO LOZOYA (SIERRA DE GUADARRAMA, ESPAÑA)
}

\author{
C. Casado, ${ }^{1}$ C. Montes, ${ }^{1}$ D. García de Jalón ${ }^{2}$ y O. Soriano ${ }^{3}$ \\ 1. Departamento de Ecología, Facultad de Ciencias. Universidad Autónoma de Madrid. Cantoblanco, 28049 Ma- \\ drid. Spain. \\ 2. Departamento de Zoología, E.T.S.I.M. Univ. Politécnica de Madrid. Spain. \\ 3. Departamento de Invertebrados, Museo Nacional de Ciencias Naturales. C/. Gutiérrez Abascal, 2. 28006 Ma- \\ drid. Spain.
}

Palabras clave: Lozoya river, stream benthos, faunistic composition, biogeographical analysis and historical factors.

\begin{abstract}
CONTRIBUTION TO THE STUDY OF THE BENTHIC FAUNA OF THE LOZOYA RIVER (GUADARRAMA RIDGE, SPAIN)
\end{abstract}

The faunistic composition of principal aquatic insect taxa which belong to the fluvial macrobenthos of the Lozoya river was studied. Four series of benthic seasonal samples were taken during 1980-81. Some observations are made concerning the distribution of the most relevant species and biogeographical analysis was undertaken to detect the historical factors characterizing their communities. A large number of different species have been identified among the lotic fauna. Many of these species sheltered in this basin in different geological periods and a lot of them have been placed in the prepleistocene age. The fluvial fauna show an important number of species with a very limited distribution, some of them being practically confined to Spain. For this reason this watershed perfom a sort of reserve in species with great environmental value.

\section{INTRODUCCIÓN}

El río Lozoya es la principal cuenca de abastecimiento de agua potable a Madrid, y cuenta en la actualidad con cinco embalses en funcionamiento, lo que ha originado una importante modificación en la composición y estructura de sus comunidades de organismos acuáticos. principalmente en sus tramos medio y bajo. Por otro lado, su cuenca, enclavada en la mitad norte de la Sierra de Guadarrama, constituye una de las zonas más singulares del centro de la Península Ibérica debido a su antigüedad, ya que se trata de uno de los núcleos permanentemente emergidos desde mediados del Paleozoico. A pesar de todo ello, se le ha prestado poca atención desde un punto de vista limnológico y los estudios realizados en su cuen-

Limnetica, 6: 87-100 (1990)

(C) Asociación Española de Limnología, Madrid, Spain ca además de ser escasos, tocan exclusivamente aspectos muy parciales; la mayoría se centran en el estudio de sus embalses: MADRID Moreno (1911), Margalef et al. (1976), Revenga (1979), Lobón (1979), Urbistondo (1980), Román et al. (1981) y HAERING (1984). En primer lugar, SCHMID (1952) recogió insectos acuáticos en los tramos superiores de cabecera. Posteriormente, GARCía DE JALón (1979) realiza un inventario de las comunidades bentónicas de macroinvertebrados del tramo alto y medio del río Lozoya, con especial atención al orden Trichoptera. GonzÁLEz DEL TÁNAGO et al. (1979) aplica diversos índices biológicos de calidad a las aguas de estos tramos fluviales. GARCÍA DE JALÓN (1980, 1982 a y b) resume los efectos del embalse de la Pinilla sobre las comunidades fluviales y posteriormente revisa las especies del orden Trichoptera para este río y establece una zonación de acuerdo con las principales comuni- 
dades macrobentónicas (GARCía DE JALON \& GONZÁlez dEl TánAgo, 1982). Finalmente, CASAdo (1986) analiza las comunidades de macroinvertebrados del eje principal del río Lozoya haciendo especial hincapié en el efecto de la regulación de sus caudales.

Este artículo presenta una lista comentada de las especies de macroinvertebrados acuáticos que componen la fauna fluvial del habitat erosional (en el sentido de Cummins, 1972) del eje principal del río Lozoya. La elección de este tipo de ambientes nos permite recoger la fauna típicamente fluvial o reófila, desechando las poblaciones de zo- nas marginales de orilla y charcas adyacentes, aunque ocasionalmente se han recogido especies intermedias o ligadas a la vegetación, debido a la escasez de caudales circulantes en determinados tramos.

\section{MATERIAL Y MÉTODOS}

El estudio ha constado de cuatro campanas de muestreo, realizadas de forma estacional durante el período 1980-81.

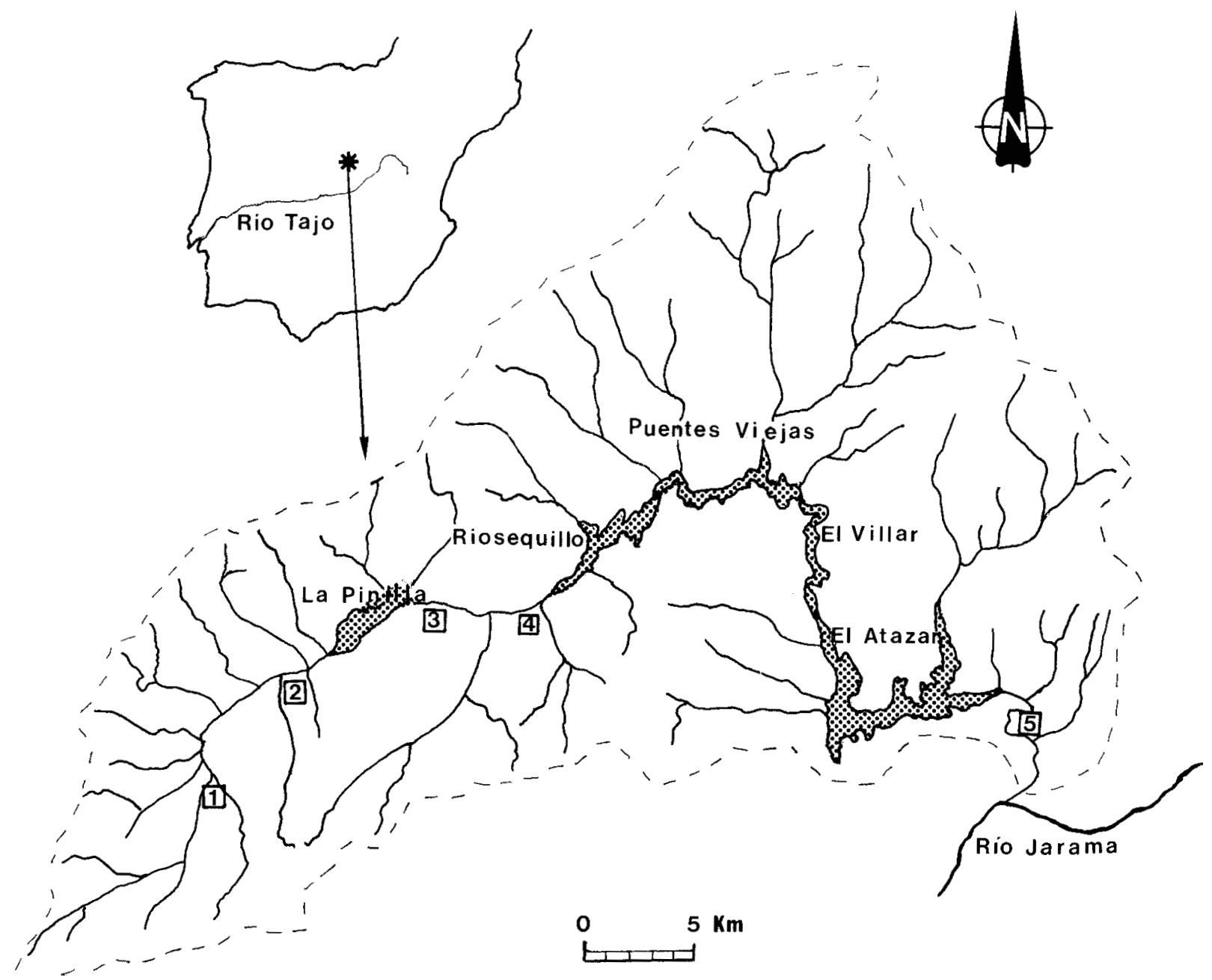

Figura 1.- Situación de los puntos de muestreo en el no Lozoya para el presente estudio. Situation of sampling stations in the Lozoya nver. 
Los organismos se recogieron empleando procedimientos tanto de carácter cualitativo (red de mano de $20 \mathrm{~cm}$ de diámetro con una luz de malla de 0,27 mm) como semicuantitativos (Schroeder's Stone lifting). Estos métodos han sido explicados detalladamente en multitud de ocasiones (ver, por ejemplo, HyNEs 1970).

En total se consideraron 5 estaciones de muestreo a lo largo del eje principal del río Lozoya, dos de ellas (1 y 2) en un sector de cabecera de $21 \mathrm{~km}$ de longitud, aguas arriba del embalse de La Pinilla, otras dos ( $\mathbf{3}$ y 4$)$ en un tramo medio de unos $7 \mathrm{~km}$ comprendido entre la presa de dicho embalse y la cola del embalse de Riosequillo, y otra (estación 3) en el último tramo lótico, de unos $5 \mathrm{~km}$ de longitud, comprendido entre la presa del Atazar y su desembocadura en el río Jarama (fig. 1).

A continuación se describen brevemente las características ambientales de cada uno de los puntos de muestreo considerados (tabla 1):

Estación 1. Presenta un cauce muy encajado y relativamente estrecho, con una elevada granulometría, principalmente grandes afloramientos de granito y gneis y alta velocidad de corriente. Por ello, la movilidad del sustrato es muy baja y las acumulaciones de sedimentos son escasas. Se trata de aguas muy frías (temperatura media anual $=6,2^{\circ} \mathrm{C}$ ), de mineralización baja (conductividad media anual $=19,7 \mu \mathrm{S})$, de $\mathrm{pH}$ bajo (media anual $=6,6$ ) y valores de oxígeno disuelto elevados (media anual $=10,6 \mathrm{mg} / \mathrm{l}$ ).

Estación 2. Está situada en un valle muy tendido, con un cauce ancho y poco profundo. La textura del sustrato es de menor tamaño que la del tramo anterior, por lo que aumenta su movilidad. Por otro lado, la presencia de sedimentos y ma- crófitos de orilla es mayor, especialmente en la época de verano, cuando disminuye el caudal. La temperatura aumenta notablemente debido a la escasa profundidad (media anual $=9,9{ }^{\circ} \mathrm{C}$ ), pero la mineralización sigue siendo muy baja (conductividad media anual $=51,2 \mu S$ ), aunque aumenta ligeramente el $\mathrm{pH}$ (media anual $=7,4$ ).

Estaciones 3 y 4. Están localizadas en el tramo inferior a la presa de la Pinilla, por lo que el efecto de la regulación se deja sentir en una serie de características. Presentan una mayor constancia estaciona1 de caudales (CASADO, 1986), por lo que se trata del tramo más profundo de todo el río. Esto origina un aumento en la estabilidad del lecho, que favorece la colonización de la vegetación riparia, por lo que, principalmente en la época de verano, la presencia de una densa vegetación de macrófitas caracteriza ambas estaciones de muestreo. Son aguas más frías de lo que les correspondería debido a los aportes de fondo del embalse (temperatura media anual $=7,6$ y $9,2{ }^{\circ} \mathrm{C}$, respectivamente), con una baja mineralización (conductividad media anual $=68,5$ y $63,7 \mu \mathrm{S}$ ), ligeramente básicas ( $\mathrm{pH}$ media anual $=8,1$ y 8,4 ) y con elevadas concentraciones de oxígeno en disolución (media anual $=9,6$ y $10 \mathrm{mg} / \mathrm{l}$ ).

Estación 5. Esta estación se caracteriza por presentar un sustrato de grano fino y una velocidad de corriente baja. Su cauce es estrecho y poco profundo, con baja movilidad del sustrato debido al déficit de caudales (ya que no se realizan sueltas desde el embalse del Atazar). Las orillas y el cauce están fuertemente colonizadas por una densa vegetación de algas bentónicas y macrófitas acuáticos y existe una importante acumulación de sedimentos finos. Se trata de aguas templadas o cálidas (temperatura media anual $=4,5^{\circ} \mathrm{C}$ ) con niveles de mineralización suaves o medianos (con-

Tabla 1.- Características fisiográficas generales de las estaciones. Descnptions of general physiographical charactenstics of sampling stations.

\begin{tabular}{cccccccc}
\hline $\begin{array}{c}\text { Estaciones } \\
\text { de muestreo }\end{array}$ & $\begin{array}{c}\text { Coordenadas } \\
\text { UTM }\end{array}$ & $\begin{array}{c}\text { Altitud } \\
(\boldsymbol{m})\end{array}$ & $\begin{array}{c}\text { Distancia al } \\
\text { origen }(\mathrm{km})\end{array}$ & $\begin{array}{c}\text { Pendiente } \\
(\%)\end{array}$ & $\begin{array}{c}\text { Anchura } \\
(\boldsymbol{m})\end{array}$ & $\begin{array}{c}\text { Profundidad } \\
\text { media }(\mathrm{cm})\end{array}$ & $\begin{array}{c}\text { Tipo de } \\
\text { sustrato }\end{array}$ \\
\hline 1 & 30T VL 256 243 & $\mathbf{1 2 3 5}$ & 12,7 & 38,63 & 13,9 & 28,1 & Grandes bloques \\
$\mathbf{2}$ & 30T VL 291 298 & $\mathbf{1 1 0 4}$ & 20,5 & 9,26 & 25,2 & 32,0 & Gravas y cantos rodados \\
3 & 30T VL 361 381 & $\mathbf{1 0 7 5}$ & 30,5 & 4,54 & 21,5 & 90,0 & Bloques y cantos rodados \\
$\mathbf{4}$ & 30T VL 399 329 & $\mathbf{1 0 3 0}$ & 35,2 & 4,54 & 23,56 & $\mathbf{8 5 , 0}$ & Grandes bloques y cantos rodados \\
$\mathbf{5}$ & 30T VL 632 257 & $\mathbf{7 0 0}$ & 90,0 & 4,00 & 11,64 & 26,3 & Cantos rodados y gravas \\
\hline
\end{tabular}


ductividad media anual $=241,2 \mu \mathrm{S})$ y ligeramente alcalinas ( $\mathrm{pH}$ media anual $=8,7$ ).

\section{RESULTADOS}

En total se recogieron 54516 lamas y 3227 pupas de 196 taxones del macrobentos fluvial del río Lozoya. Estos taxones se agrupan en 142 especies de 95 géneros pertenecientes a 11 órdenes distintos de macroinvertebrados acuáticos. Sin embargo, el estudio sistemático se ha centrado exclusivamente en los grupos más importantes presentes en las muestras, cuyos resultados se resumen en la tabla 2, recogiendo así, un total de 150 especies pertenecientes a 10 órdenes, más 29 taxones de la familia Chironomidae, en la cual ha sido imposible llegar a un nivel taxonómico inferior al de género, debido a las enormes dificultades que presenta la determinación a nivel específico de las larvas de este grupo.

Como puede apreciarse en el análisis de los datos presentados en la tabla 2, tres son los órdenes que tienen un mayor peso en la composición específica de las comunidades, y están presentes en todas las estaciones de muestreo: Trichoptera (84 especies), Diptera (37 taxones) y Ephemeroptera (24 especies). Respecto a los plecópteros, su riqueza de especies es importante únicamente en las estaciones superiores, de cabecera (E-1 y E-2), mientras que en las afectadas por el efecto de regulación del caudal, aguas abajo de Pinilla desaparecen por completo, si bien se recolectan algunas especies en el tramo final (E-5). El resto de los grupos (heterópteros, coleópteros, odonatos, moluscos, hirudíneos y crustáceos) presentan una riqueza de especies considerablemente menor y se encuentran en general mejor representados en los tramos bajos.

\section{Trichoptera}

Se han capturado un total de 84 especies pertenecientes a 46 géneros, de las cuales 65 se encontraban ya citadas para este río por GARCía DE JALON (1979). Entre la fauna de tricópteros existe un gran número de endemismos peninsulares: 9 especies endémicas de la Península Ibérica (Rhyacophila adjunta, R. terpsichore, Glossosoma privatum, Hydropsyche dubia, $\mathrm{H}$. pictetorum, $\mathrm{H}$. tibialis, Psychomyia ctneophora, Larcasia partita y Ceraclea sobradielis), 2 de la Península Ibérica y Norte de África (Hydropsyche lobata, Schizopelex festiva), 1 de la Península Ibérica y Pirineos (Ryacophila relicta), 1 de la Sierra de Guadarrama (Allogamus laureatus) y 1 de Córcega y la Sierra de Guadarrama (Agapetus cyrnensis segovicus). El género Larcasia representa una disyunción interesante, ya que su distribución se restringe a la Península Ibérica y a la vertiente sur del Himalaya (DA Terra, 1981; González et al., 1987). El resto de especies de tricópteros encontradas presentan distribuciones más amplias.

\section{Diptera}

Dos familias, simúlidos y quironómidos, constituyen un elemento constante en casi todas las muestras de bentos fluvial recogidas en el río Lozoya en todas las épocas del año.

De la familia Simuliidae se han llegado a identificar únicamente 8 especies, pertenecientes a 5 géneros y subgéneros: 1 especie de Prosimulium (P. [P.] hirtipes-tomosvaryi), 1 especie del subgénero Eusimulium y otra de Nevermannia, de amplia distribución por toda la región holártica (S. [E.] gr. aureum y S. (N.) vernum), 3 especies de Wilhelmia de distribución euroasiática incluyendo África del Norte (S. [W.] equinum, S. [W./ lineatum y S. [W.] pseudoequinum), y 2 especies de Simulium bien distribuidas en la región Paleártica y Oriental (S. [S.] ornatum) y (S. [S.] argyreatum). (Puig et al., 1984; González et al., 1987).

De la familia Chironomidae se han identificado un total de 29 taxones (tabla 2). Esta familia constituye un grupo de dípteros muy antiguo, que ya llegó a un alto nivel evolutivo en el periodo Jurásico (FITTKaU, 1986), por lo que en esta época posiblemente ya existían la mayoría de los géneros del grupo. Esto, junto con su fácil dispersión debida al vuelo, implica el cosmopolitismo de un gran número de sus géneros. La región holártica presenta 74 géneros de los que 54 son comunes a las regiones paleártica y neártica, lo cual refleja el elevado cosmopolitismo del grupo (AsHe et al., 1987). Ninguno de los géneros exclusivos de la región paleártica está presente en el río Lozoya. Así pues, los géneros tratados en este trabajo presentan una clara distribución holártica (REIss, 1978). Es previsible que el estudio de exuvias pupales o adultos, permita alcanzar el nivel específico en la 
Tabla 2.- Composición faunística de las comunidades de macrobentos del eje principal del no Lozoya

Faunistic composition of macrobenthic communities in the principal axe of the Lozoya nver.

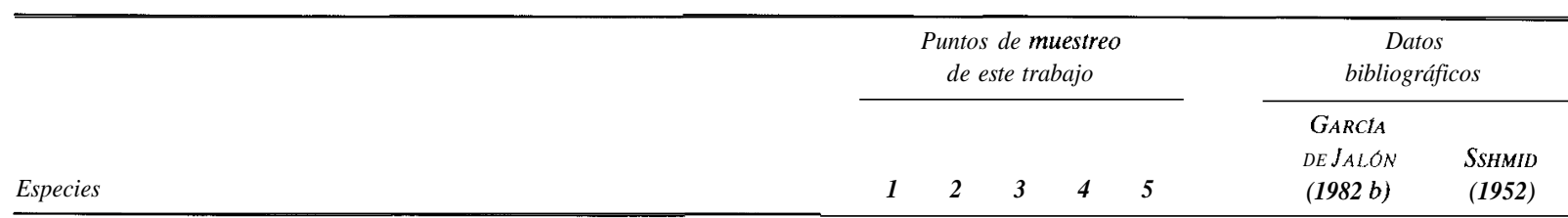

MOLLUSCA

Lymnaea auricularia Linnaeus 1758

L. peregra Müller 1774

Physella acuta Draparnaud 1805

Gyraulus albus Müller 1774

Planorharius corneus Linnaeus 1758

Ancylus fluviatilis Müller 1774

Pisidium sp.

ANNELIDA HIRUDINEA

Glossiphonia complanata L. 1768

Helobdella stagnalis L. 1758

Batracobdella paludosa Carena 1824

Haemopis sanguisuga Linnaeus 1758

Erpobdella monostriata (Gedroyc. 1916)

$\begin{array}{lllll}\cdot & \cdot & & & + \\ + & + & + & + & . \\ \cdot & \cdot & \cdot & \cdot & + \\ \cdot & \cdot & + & + & + \\ + & + & + & \cdot & . \\ + & + & + & + & + \\ . & + & + & . & .\end{array}$

\section{CRUSTACEA DECAPODA}

Atyaephyra desmarestii Millet 1831

\section{INSECTA EPHEMEROPTERA}

Siphlonurus lacustris Eaton 1870

Baetis fuscatus (L. 1761)

B. melanonyx Pietet 1843-45

B. muticus Linnaeus 1758

B. cf. pavidus Grandi 1949

B. rhodani Pictet 1843

Centroptilum luteolum (Müller 1776)

C. pennulatum Eaton 1870

Cloeon dipterum (Linnaeus 1761)

Oligoneuriella rhenana Imhoff 1852

Epeorus cf. torrentium Eaton 1881

Rhithrogena semicolorata Curtis 1834

Ecdyonurus venosus Fabricius 1775

Heptagenia sulphurea Müller 1776

Ephemerella ignita (Poda 1761)

Serratella hispanica Eaton 1887

Drunella paradinasi Tanago \& Jalon 1983

Caenis luctuosa (Burmeister 1839)

Calliarcys humilis Eaton 1881

Choroterpis picteti (Eaton 1871)

Leptophlebia vespertina Linnaeus 1746

Paraleptophlebia submarginata (Stephens 1835)

Habrophlebia fusca (Curtis 1834)

H. nervulosa Eaton 1883-88

\section{INSECTA PLECOPTERA}

Brachyptera arcuata (Klapálek 1902)

Amphinemura sulcicollis guadarramensis Aubert 1952

Nemoura fulviceps Klapalek 1902

Protonemura intricata iberiaca Zwick 1962 


\begin{tabular}{|c|c|c|c|c|c|c|c|}
\hline \multirow[b]{2}{*}{ Especies } & \multicolumn{5}{|c|}{$\begin{array}{l}\text { Puntos de muestreo } \\
\text { de este trabajo }\end{array}$} & \multicolumn{2}{|c|}{$\begin{array}{c}\text { Datos } \\
\text { bibliográficos }\end{array}$} \\
\hline & 1 & 2 & 3 & 4 & 5 & $\begin{array}{c}\text { GARCla } \\
\text { DE JALON } \\
(1982 \boldsymbol{b})\end{array}$ & $\begin{array}{l}\text { SСнмID } \\
(1952)\end{array}$ \\
\hline P. meyeri (Pictet 1841) & + & + & . & . & + & & \\
\hline P. pyrenaica asturica Aubert 1954 & + & . & . & . & . & & \\
\hline Leuctra geniculata Stephens 1835 & . & . & . & . & + & & \\
\hline L. hippopus Kempny 1899 & + & + & . & . & + & & \\
\hline Capnia nigra (Pictet 1833) & + & + & . & . & + & & \\
\hline Isoperla curtata Navas 1924 & . & + & . & . & + & & \\
\hline Perlodes microcephala (Pictet 1833) & + & + & . & . & . & & \\
\hline Dinocras cephalotes (Curtis 1827) & + & + & . & . & . & & \\
\hline Chloroperla tripunctata (Scopoli 1763) & . & . & . & . & + & & \\
\hline Siphonoperla torrentium (Pictet 1841) & + & + & . & . & . & & \\
\hline
\end{tabular}

\section{INSECTA ODONATA}

Calopteryx haemorrhoidalis (Van der Linden 1825)

Platycnemis acutipennis Selys 1841

Coenagrion mercuriale (Charpentier 1840)

Onychogomphus uncatus (Charpentier 1840)

\section{INSECTA HETEROPTERA}

Hydrometra stagnorum (Linnaeus 1758)

Mesovelia vittigera Horvath 1895

Velia caprai caprai Tamanini 1947

Aphelocheirus aestivalis aestivalis Fabricius 1794

Micronecta meridionalis (Costa 1860)

Naucoris maculatus maculatus Fabncius 1798

Nepa cinerea Linnaeus 1758

\section{INSECTA COLEOPTERA}

Haliplus lineaticollis (Marsham 1802)

Colymbetes fuscus (Linnaeus 1758)

Dytiscus pisanus Castelnau 1834

Orectochilus villosus (Müller 1776)

Hydraena inapicipalpia Pictet 1842

Dupophilus brevis Mulsant \& Rey 1872

Elmis aenea (Müller 1806)

Limnius opacus Müller 1806

\section{INSECTA TRICHOFTERA}

Rhyacophila adjunta MacL. 1884

R. dorsalis Curtis 1834

R. lusitanica MacL. 1884

R. meridionalis Pictet 1866

R. mocsaryi tredosensis Schmid 1952

R. munda MacL. 1862

R. obliterata MacL. 1879

R. occidentalis MacL. 1979

R. pulchra Schmid 1952

$R$. relicta MacL. 1884

R. terpsichore Malicky 1976

R. tristis Pictet 1834

Glossosoma privatum MacL. 1884

Agapetus cyrnensis segovicus Mosely 1930

A. laniger Pictet 1834

Agraylea sp. Curtis 1834 


\begin{tabular}{|c|c|c|c|c|c|c|c|}
\hline \multirow[b]{2}{*}{ Especies } & \multicolumn{5}{|c|}{$\begin{array}{l}\text { Puntos de muestreo } \\
\text { de este trabajo }\end{array}$} & \multicolumn{2}{|c|}{$\begin{array}{c}\text { Datos } \\
\text { bibliográficos }\end{array}$} \\
\hline & 1 & 2 & 3 & 4 & 5 & $\begin{array}{c}\text { GARCIA } \\
D E J_{A L O N} \\
(1982 b)\end{array}$ & $\begin{array}{c}\text { SCHMID } \\
(1952)\end{array}$ \\
\hline Hydroptila veclis Curtis $\mathbf{1 8 3 4}$ & . & . & . & . & + & & \\
\hline Ithythrichia sp. & . & . & + & . & . & & \\
\hline Orthothrichia sp. & . & . & . & + & + & & \\
\hline Oxyethina falcata Morton $\mathbf{1 9 8 3}$ & . & . & . & . & + & & \\
\hline Ptilocolepus extensus MacL. 1884 & . & . & . & . & . & & \\
\hline Stactobia furcata Mosely $\mathbf{1 9 3 0}$ & . & . & . & . & + & + & \\
\hline Allotrichia marikovicae Malicky 1977 & $\cdot$ & . & . & . & , & + & \\
\hline Phylopotamus amphilectus MacL. 1884 & + & . & . & . & & + & \\
\hline P. montanur Donovan 1813 & & & & & & + & + \\
\hline Wormaldia triangulifera beaumonti Schmid 1952 & & & & & & + & + \\
\hline W. variegata mattheyi Schmid 1952 & & & & & & + & + \\
\hline Cheumatopsyche lepida (Pictet 1834) & & & & + & + & & + \\
\hline Hydropsyche ambigua Schmid 1952 & & & & & & & + \\
\hline H. bulbifera MacL. 1878 & . & . & . & . & + & & + \\
\hline H. cf. dinarica Marinkovic 1979 & + & . & . & . & + & & \\
\hline H. dubia Navas 1932 & + & + & . & + & + & & \\
\hline H. exocellata Dufour 1841 & + & + & . & + & + & & . \\
\hline H. lobata MacL. 1884 & . & . & . & . & + & & + \\
\hline H. pellucidula Curtis 1834 & + & + & + & + & + & & + \\
\hline H. pictetorum Botosaneau \& Schmid 1973 & - & + & . & . & + & & \\
\hline H. siltalai Dohler 1963 & + & + & + & + & + & & \\
\hline H. tibialis MacL. 1884 & + & + & . & . & + & & + \\
\hline Plectrocnemia conspersa (Curtis 1834) & . & + & + & + & . & & + \\
\hline P. geniculata MacL. 1884 & . & + & . & . & . & & . \\
\hline Polycentropui flavomaculatus (Pictet 1834) & + & + & + & + & + & & + \\
\hline P. kingi MacL. 1881 & + & . & . & . & + & & + \\
\hline P. telifer MacL. 1884 & . & . & . & . & . & & + \\
\hline Psychomyia ctneophora MacL. 1884 & - & + & + & . & + & & + \\
\hline P. pusilla (Fabricius 1781) & . & + & + & + & + & & + \\
\hline Tinodes antequeruela Schmid 1954 & . & + & + & . & + & & \\
\hline T. waeneri (Linnaeus 1758) & . & . & . & . & . & & \\
\hline Ecnomus sp & . & . & . & . & + & & \\
\hline Beraea sp & . & . & + & . & $\cdot$ & & \\
\hline Oligoplectrum maculatum (Fourcroy 1785) & + & . & . & . & . & & + \\
\hline Micrasema cinereum Mosely 1930 & . & . & . & . & . & & + \\
\hline M. longulum MacL. 1876 & + & + & . & . & . & & + \\
\hline M. moesta (Hagen 1868) & + & + & . & . & . & & + \\
\hline Anomalopterygella chauviniana (Stein 1874) & + & + & . & + & . & & + \\
\hline Limnephilus auricola Curtis $\mathbf{1 8 3 4}$ & & & & & & & + \\
\hline L. bipunctatus Curtis $\mathbf{1 8 3 4}$ & & & & & & & + \\
\hline L. politus MacL. 1865 & & & & & & & + \\
\hline L. vittatus Fabncius 1798 & & & & & & . & + \\
\hline L. rhombicus (Linnaeus 1758) & . & . & + & . & . & . & \\
\hline Anabolia nervosa Curtis $\mathbf{1 8 3 4}$ & + & $\cdot$ & + & + & . & . & + \\
\hline Potamophylax latipennis (Curtis 1834) & + & + & . & . & . & . & + \\
\hline Halesus radiatus Curtis 1834 & . & + & . & . & . & & + \\
\hline Stenophylax vibex Curtis $\mathbf{1 8 3 4}$ & . & + & + & + & . & & + \\
\hline Mesophylax aspersus (Rambur 1842) & . & . & . & . & + & & \\
\hline Allogamui laureatus (Navas 1918) & . & + & . & + & . & & + \\
\hline A. ligónifer (MacL. 1876) & + & + & + & . & . & & + \\
\hline Melampophylax sp. & + & . & . & . & . & & \\
\hline Chaetopteryx villosa Fabricius $\mathbf{1 7 9 8}$ & + & + & + & + & + & & + \\
\hline Silo graellsii Pictet $\mathbf{1 8 6 5}$ & & & & & & & + \\
\hline Larcasia partita Navas 1917 & & & & & & & + \\
\hline Thremma gallicum (MacL. 1880) & & & & & & & + \\
\hline
\end{tabular}




\begin{tabular}{|c|c|c|c|c|c|c|c|}
\hline \multirow[b]{2}{*}{ Especies } & \multicolumn{5}{|c|}{$\begin{array}{l}\text { Puntos de muestreo } \\
\text { de este trabajo }\end{array}$} & \multicolumn{2}{|c|}{$\begin{array}{c}\text { Datos } \\
\text { bibliográficos }\end{array}$} \\
\hline & $I$ & 2 & 3 & 4 & 5 & $\begin{array}{c}\text { GARCtA } \\
\text { DE JALÓN } \\
(1982 b)\end{array}$ & $\begin{array}{l}\text { SCHMID } \\
(1952)\end{array}$ \\
\hline T. tellae Gonzalez 1978 & . & . & . & . & . & . & + \\
\hline Lepidostoma hirtum (Fabricius 1775) & & . & . & . & . & + & + \\
\hline Crunoecia irrorata (Curtis 1834) & + & . & . & . & . & . & + \\
\hline Lasiocephala basalis Kolenati 1848 & . & + & . & . & . & . & . \\
\hline Athripsodes bilineatus (Linnaeus 1758) & . & . & . & . & & . & + \\
\hline A. braueri (Pictet 1865$)$ & . & + & . & . & . & + & + \\
\hline Ceraclea sobradielis (Navas 1917) & . & . & . & + & . & . & + \\
\hline Leptocerus lusitanicus & + & & + & . & & 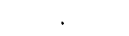 & \\
\hline Mystacides azurea (Linnaeus 1761) & . & . & . & . & . & . & + \\
\hline Adicella reducta MacL. 1865 & . & . & . & . & . & + & + \\
\hline Schizopelex festiva (Rambur 1842) & + & & & . & & + & + \\
\hline Sericostoma baeticum Pictet 1885 & . & . & . & . & . & . & + \\
\hline S. selysii Pictet 1865 & + & + & + & + & · & . & + \\
\hline \multicolumn{8}{|l|}{ INSECTA DIPTERA (SIMULIIDAE) } \\
\hline Prosimulium $(P$.$) hirtipes-tomosvaryi$ & + & + & . & & & & \\
\hline Simulium (Eusimulium) gr. aureum & . & + & + & + & + & & \\
\hline S. (Nevermannia) vernum Macquant 1826 & + & + & . & . & . & & \\
\hline S. (Wilhemia) equinum (Linnaeus 1758) & . & + & + & + & . & & \\
\hline S. (W.) lineatum Meigen 1804 & . & . & + & & + & & \\
\hline S. (W.) pseudequinum Séguy 1921 & . & . & + & . & + & & \\
\hline S. (Simulium) ornatum Meigen 1818 & + & + & + & + & + & & \\
\hline S. (S.) argyreatum Meigen 1838 & + & . & + & & & & \\
\hline \multicolumn{8}{|l|}{ INSECTA DIPTERA (CHIRONOMIDAE) } \\
\hline Diamesa sp. Meigen 1938 & . & . & + & + & + & & \\
\hline Potthastia sp. Kieffer 1922 & + & + & + & + & + & & \\
\hline Pseudodiamesa sp. Goetghebuer 1939 & + & & & & . & & \\
\hline Syndiamesa sp. Kieffer 1918 & . & + & + & & . & & \\
\hline Sympotthastia sp. Pagast 1947 & . & + & + & . & . & & \\
\hline Paramerina sp. Fittkau 1962 & + & . & . & & . & & \\
\hline Thienemannimyia gr. Fittkau 1962 & . & + & + & + & + & & \\
\hline Brillia sp. Kieffer 1913 & $\cdot$ & + & + & . & . & & \\
\hline Corynoneura sp. Winnertz 1846 & + & . & & + & + & & \\
\hline Cricotopus-Orthocladius spp. Van der Wulp 1874 & + & + & + & + & + & & \\
\hline Eukiefferiella sp. Thienemann 1926 & + & + & + & + & + & & \\
\hline Nanocladius sp. Kieffer 1913 & $\cdot$ & $\cdot$ & + & + & + & & \\
\hline $\begin{array}{l}\text { Psectrocladius sp. Kieffer } 1906 \\
\text { Rheocricotopus sp. Thienemann \& Harnish } 1932\end{array}$ & + & 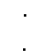 & + & + & + & & \\
\hline Synorthocladius sp. Thienemann 1935 & & + & + & + & + & & \\
\hline Thienemanniella sp. Kieffer 1911 & + & + & + & + & + & & \\
\hline Chironomus gr. thummi Kieffer 1911 & . & . & . & + & . & & \\
\hline Endochironomus sp. Kieffer 1918 & . & & + & . & . & & \\
\hline Microtendipes sp. Kieffer 1915 & + & . & + & + & + & & \\
\hline Pentapedilum sp. Kieffer 1913 & . & . & + & + & . & & \\
\hline Polypedilum gr. convictum Walkcr 1856 & $\cdot$ & + & + & + & . & & \\
\hline P. gr. nubeculosum Meigen 1818 & $\dot{+}$ & i & + & + & $\cdot$ & & \\
\hline P. gr. scalaenum Schrank 1803 & + & $\begin{array}{l}+ \\
+\end{array}$ & + & + & + & & \\
\hline $\begin{array}{l}\text { Stictochironomus sp. Kieffer } 1919 \\
\text { Cladotanytarsus sp. Kieffer } 1921\end{array}$ & . & + & + & + & $\begin{array}{l}+ \\
+\end{array}$ & & \\
\hline Micropsectra sp. Kieffer 1911 & + & + & + & + & + & & \\
\hline Paratanytarsus sp. Thienemann \& Bause 1913 & & . & & + & . & & \\
\hline Rheotanytarsus sp. Bause \& Thienemann 1913 & + & + & + & + & + & & \\
\hline Tanytarsus sp. Van der Wulp 1876 & . & + & + & + & + & & \\
\hline
\end{tabular}


determinación y muestre la existencia de especies incluso no descritas y/o endémicas de la Península Ibérica.

\section{Ephemeroptera}

Se han reconocido un total de 24 especies de efemerópteros pertenecientes a 18 géneros; las familias Baetidae, con 8 especies, y Leptophlebiidae, con 6 especies, son las más representadas. Entre las especies citadas (tabla 2), se encontraron únicamente 3 restringidas a la Península Ibérica (Serratella hispanica, Drunella paradinasi, y Calliarcys humilis), mientras que el resto tienen una distribución mucho más amplia. Es de destacar que los géneros Drunella y Serratella, confinados exclusivamente al extremo noroeste de la Península Ibérica para Europa, se encuentran muy bien representados en la región neártica y habitan también en Siberia oriental, lo que representa una disyunción biogeográfica anfiatlántica (BREHM, 1947) muy peculiar.

\section{Plecoptera}

Se han identificado un total de 14 especies, pertenecientes a 11 géneros distintos. Entre ellas se pueden distinguir 5 especies y subespecies peninsulares (Brachyptera arcuata, Amphinemura sulcicollis guadarramensis, Protonemura intricata iberiaca, Protonemura pyrenaica asturica e Isoperla curtata), mientras que las restantes especies se encuentran presentes también en amplias áreas europeas.

El esto de los órdenes presentan distribuciones muy amplias (Mollusca, Hirudinea, Crustacea y Odonata) y/o principalmente europeas y mediterráneas (Heteroptera y Coleoptera), con muy pocas especies relevantes.

\section{DISCUSIÓN}

Si se compara el número de especies encontradas para cada uno de estos órdenes en el río Lozoya con el número encontrado en otros ríos de la Península Ibérica, se puede tener idea de su elevada riqueza relativa de especies (PRAT et al., 1983; Puig et al., 1984; García de Jalón \& GonZÁlez del Tánago, 1986, entre otros). Por otro lado, la importancia de su fauna no es debida exclusivamente al elevado número de especies, ya que podría tratarse de una fauna banal, cosmopolita o de escasa relevancia. Sin embargo, esto no ocurre, ya que existe un elevado número de especies endémicas, con distribuciones muy restringidas o particularmente notables.

La fauna que compone el bentos típicamente fluvial (recogida exclusivamente en el hábitat erosional) del eje principal del río Lozoya tiene los siguientes tipos de distribución:

\section{a) Elementos hespéricos}

Son endemismos ibéricos que se distribuyen principalmente por los sistemas montañosos del cuadrante nororiental de la Península Ibérica y Sistema Central (Berthelemy \& Whytton DA Terra, 1980). Coinciden con los denominados textualmente por AUBERT (1963) «nordibériques». Estos sistemas montañosos pueden haber servido de refugio evolutivo a muchas especies estenotermas de aguas corrientes y poco mineralizadas, ya que constituyen el núcleo más antiguo de tierras permanentemente emergidas desde mediados de la era Paleozoica (MelÉndez, 1958), no conectado con el resto de la Península Ibérica hasta finales del Mioceno, poco antes de las glaciaciones pliocenas y pleistocenas. Este tipo de distribución se caracteriza por un reciente y amplio aislamiento geográfico, con áreas de distribución muy cerradas en regiones montañosas o geológicàmente antiguas. En algunos casos tienen pares de áreas disjuntas pero pertenecen a grupos relictos o aislados sistemáticamente (como en el caso de Larcasia partita). Se trata de una fauna muy antigua que nos obliga a remontarnos a un período geológico anterior al Cuaternario. Incluímos en este grupo a las siguientes especies: Serratella hispanica, Drunella paradinasi, Calliarcys humilis, Brachyptera arcuata, Amphinemura sulcicollis guadarramensis, Protonemura intricata iberiaca, Protonemura pyrenaica asturica, Isoperla curtata, Rhyacophila adjunta, Rhyacophila terpsichore, Glossosoma privatum, Hydropsyche dubia, Hydropsyche tibialis, Psychomyia ctneophora, Larcasia partita y Ceraclea sobradielis.

b) Elementos lusitánicos

Este grupo está formado por especies que permanecieron en la Península Ibérica y las zonas de 
baja montaña de Europa Central libres de hielo durante todo el Pleistoceno, protegidas por las especiales condiciones de pendiente, velocidad de la corriente y temperatura del agua de estos ríos de baja montaña, en los que nunca se dejó sentir el efecto de las glaciaciones del Plioceno y Pleistoceno (MaLickY, 1983), y que con posterioridad se extendieron, principalmente por el extremo occidental húmedo de Europa, que constituye un corredor de condiciones ecológicas favorables (MARGalef, 1955 a y b). Son ejemplo de este tipo de especies: Baetis pavidus, Rhyacophila occidentalis, Micrasema longulum, Anomalopterygella chauviniana, Anabolia nervosa, Mesophylax aspersus, Allogamus ligonifer, Leptocerus lusitanicus, Schizopelex festiva y Simulium (Nevermannia) vernum. Algunas especies lusitánicas tienen una distribución restringida a la Península Ibérica y poco más allá de los Pirineos (es el caso de $\mathrm{Du}$ pophilus brevis, Rhyacophila meridionalis, $R$. guadarramica y $\boldsymbol{R}$. relicta), mientras que otras se encuentran también en el Norte de África, tales como Leuctra geniculata, Calopteryx haemorroidalis, Dytiscus pisanus, Hydraena inapicipalpia, Rhyacophila munda, Hydroptila veclis, Hydropsyche lobata y Schizopelex festiva.

Respecto a sus exigencias ecológicas, estas especies constituyen un grupo muy heterogéneo y presentan amplios márgenes en sus principales requerimientos, como temperatura, oxígeno y nutrientes. En general, se trata de especies de grupos antiguos con distribuciones restringidas, principalmente a la Península Ibérica y Norte de África, y a veces en pequeñas áreas de Europa sudoccidental, siendo raras o encontrándose ausentes en la mayor parte de Europa. A veces presentan un segundo centro de distribución en el sudoeste europeo o en Asia Central. Es el caso de Rhyacophila occidentalis o Dupophilus brevis, constituyendo de esta forma un claro ejemplo de disyunción este-oeste.

Las especies lusitánicas de macroinvertebrados se encuentran bien representadas en los ríos montañosos de la Península (Bertrand, 1966). Ocurre lo mismo con las especies de microinvertebrados; así las especies lusitánicas son, junto con las atlánticas, las principales acompañantes de las boreo-alpinas en las comunidades de zooplancton de lagos de alta montaña peninsulares (MIRACLE, 1982).
Sin embargo, los ríos montañosos pueden considerarse según MALICKY (1983) como biotopos extrazonales tropicales desde un punto de vista fisiológico y ecológico, debido a sus menores rangos de variación de temperaturas y a la aportación constante de alimento que asegura una mayor constancia en las condiciones de vida (W ARD \& StANFORD, 1983; Resh et al., 1988).

Otro aspecto muy importante es la posibilidad de desplazamientos verticales de acuerdo con los cambios climáticos. En el rango de temperatura de los ríos montañosos que depende, en general del clima de la región, tienen una gran incidencia las influencias locales, por lo que ríos y arroyos muy próximos presentan diferentes condiciones térmicas (MALICKY, 1983). De esta forma, los animales acuáticos pueden cambiar de río para encontrar condiciones más templadas o biotopos adecuados en un rango de cortas distancias horizontales.

\section{c) Elementos centro-sud-europeos}

Son especies de diversos orígenes que se distribuyen por sistemas montañosos de Pirineos-Cantábricos, Alpes y Cárpatos con una extensión más o menos completa por las penínsulas Ibérica, Itálica y Balcánica (AUBERT, 1963). Entre ellas hemos encontrado en el río Lozoya: Baetis melanonyx, Oligoneuriella rhenana, Epeorus torrentium, Choroterpes picteti, Nemoura fulviceps, Capnia nigra, Chloroperla tripunctata, Siphonoperla torrentium, Coenagrion mercuriale, Limnius opacus, Hydropsyche bulbifera, Plectonemia geniculata, Polycentropus kingi, Oligoplectrum maculatum y Micrasema moestum.

Estas especies presentan unas áreas de distribución que en líneas generales coinciden con las áreas centrales de dispersión, a partir de las cuales se han extendido y que les han servido de refugio durante el Pleistoceno. Según MaLicky (1983) constituyen un tipo biológico de fauna (al menos para los tricópteros) de ríos montañosos denominada «dinodal». Su fundamento ecológico es la combinación de cuatro elementos interdependientes: aguas corrientes turbulentas, tramos encajados con elevada pendiente, sustrato duro, y alta velocidad de la corriente; lo que tiene como consecuencia unos óptimos de disponibilidad de oxígeno disuelto para la fauna acuática. Por otro lado, los insectos de los ríos montañosos toleran 
temperaturas bajas del agua y no se ven necesariamente forzados a emigrar largas distancias para encontrar condiciones más templadas (desplazamientos verticales a lo largo de un eje y horizontales entre ríos y arroyos próximos), por ejemplo, durante una glaciación. Por estas razones, han podido sobrevivir y permanecer en los mismos parajes durante todo el Pleistoceno. De esta forma, el centro de origen de estos animales europeos dinodales está claramente situado en las regiones montañosas de Europa central, incluyendo el norte de la Península Balcánica. Aunque muchas especies han extendido sus áreas de distribución después de las glaciaciones al retirarse el hielo, otras no lo han hecho, de forma que el número total de especies dinodales decrece hacia el norte y el sur de Europa.

\section{d) Elementos holoeuropeos}

Especies de amplia distribución que, en general tienen un carácter eurióico. Entre otras podemos citar: Baetis fuscatus, B. muticus, B. rhodani, Ephemerella ignita, Caenis luctuosa, Paraleptophlebia submarginata, Protonemura meyeri, Leuctra hippopus, Perlodes microcephalus, Dinocras cephalotes, Plactycnemis acutipennis, Velia caprai caprai, Micronecta meridionalis, Hydropsyche pellucidula, Polycentropus flavomaculatus, Psychomyia pusilla, Stenophylax vibex.

Como se puede observar, la fauna reófila del río Lozoya está compuesta por una gran variedad de especies con diversos orígenes que coexisten perfectamente. Esto puede ser consecuencia de las condiciones favorables que ha presentado esta cuenca hidrográfica desde tiempos geológicos muy antiguos. En primer lugar, el río Lozoya está localizado en un valle elevado enclavado entre dos sistemas montañosos de gran altitud, situado en la Sierra de Guadarrama (Sistema Central). Es decir, son tierras permanentemente emergidas desde mediados de la era Paleozoica, por lo que han podido servir de refugio evolutivo a numerosas especies hespéricas. Estos elementos representan un $20,34 \%$ de la fauna de tricópteros, un $12,5 \%$ de la de efemerópteros y un 35,71\% de la de plecópteros. Por otro lado, las glaciaciones han tenido escasa repercusión debido a que la Sierra de Guadarrama presenta condiciones poco favorables para el glaciarismo, por lo que este fenóme- no ha tenido un desarrollo muy limitado, breve y muy localizado (Martínez de Pisón y López MarTínEZ, 1986). Es decir, ha permitido que numerosas especies se refugiaran en estos sistemas montañosos durante el Pleistoceno. Los elementos lusitánicos son también abundantes y representan el $20,34 \%$ de la fauna de tricópteros, $4,16 \%$ en efemerópteros y 7,14 \% en plecópteros. Además presentan las condiciones típicas de ríos de montaña, aguas de corriente turbulenta con pendientes elevadas, sustrato duro (principalmente granitos y gneis en casi toda su cuenca) y alta velocidad de corriente, lo que ha permitido que una serie de elementos de origen centroeuropeo y dinodal hayan permanecido en estos parajes durante el Pleistoceno, en algunos casos extendiendo posteriormente sus áreas de distribución. La fauna centro-sudeuropea representa el $10,17 \%$ de los tricópteros, $20,83 \%$ de los efemerópteros y $28,57 \%$ de los plecópteros. Dentro de los elementos holoeuropeos podemos encontrar un 8,47\% de los tricópteros, $33,30 \%$ de los efemerópteros y 28,57 \% de los plecópteros. El resto de elementos está constituido por especies de distribución mucho más amplia, que pueden encontrarse incluso también en otros continentes, como por ejemplo el este asiático y Norteamérica, este es el caso de algunas especies de tricópteros y efemerópteros de aguas tranquilas que ya se han comentado anteriormente.

Parece ser que la fauna del río Lozoya está compuesta por un gran número de especies que han utilizado esta cuenca hidrográfica como área de refugio y/o centro de especiación en distintos períodos geológicos, estando formada mayoritariamente por elementos asentados en ella en un período prepleistoceno, coincidiendo con MARGALEF (1949) en que las aguas dulces de las montañas del centro de España apenas han recibido elementos de los dispersados sobre el resto de Europa durante el Cuaternario.

La fauna del río Lozoya supone una reserva de especies de gran valor ambiental para la zona (provincia de Madrid), donde la mayoría de los ríos y cuerpos de agua han sufrido una fuerte degradación - e $n$ algunos casos irreversible - debido a la elevada presión demográfica y a la contaminación. Sin embargo, este río también ha soportado grandes modificaciones, debido a la desmesurada proliferación de embalses para abas- 
tecimiento en su tramo medio y bajo. En la actualidad cuenta con cinco embalses en funcionamiento, lo que supone que de sus $91 \mathrm{~km}$ de longitud, aproximadamente $58 \mathrm{~km}$ están ocupados por este tipo de cuerpos de agua. Esta situación ha originado, en estos tramos medios y bajos, un empobrecimiento de su fauna tanto por pérdida de especies (los plecópteros desaparecen total o parcialmente en las estaciones afectadas por la regulación: 3,4 y 5), como por sustitución de especies (dominancia de especies generalistas, eurioicas y cosmopolitas de bajo valor ambiental que desplazan a las reófilas regionales propias de este río y adaptadas a sus particulares condiciones). $\mathrm{La}$ pérdida sufrida por estas causas no puede ser evaluada en la actualidad debido a la inexistencia de un inventario faunístico previo a la construcción de estos embalses. Sin embargo, sí podemos suponer que ha debido ser de grandes proporciones al observar la elevada riqueza de especies que aún pueblan sus aguas y el gran valor ambiental de éstas (principalmente en sus tramos alto-medio no regulado). Por otra lado, esta pérdida de especies y diversidad ha debido ser más drástica en su tramo medio (actualmente embalsado), ya que es en esta zona donde teóricamente en ríos naturales se puede observar la mayor diversidad biótica, así como el mayor grado de heterogeneidad y variabilidad ambiental (VANNOTTE et al., 1980; MinsHALL, 1988), y donde el impacto de la regulación es mayor (STANFORD \& WARD, 1983).

\section{AGRADECIMIENTOS}

Numerosas personas han revisado desinteresadamente distintos grupos de organismos que presentaban dificultades en su determinación taxonómica; a todos ellos, queremos agradecerles su inestimable ayuda: Ignacio García Mas (Hirudinea), Rosario Vidal Abarca (Mollusca), Marta González del Tánago (Plecoptera y Ephemeroptera), M." Luisa Suarez (Odonata) y Gloria González Peña (Simuliidae).

\section{Bibliografía}

Ashe, P., D.A. Murray \& F.V. ReIss, 1987. The zoogeographical distribution of Chironomidae. (Insecta: Diptera). Annls. Limnol. 23 (1): 27-60.

Aubert, J., 1963. Les plécoptéres de la Péninsule Ibérique Eos 34: 1-107.

Berthelemy, C. \& L.W. Da Terra, 1980. Plécoptéres du Portugal. Annl. Limnol. (16) 2: 159-182.

Bertrand, H., 1966. Hemipteres, Heteropteres aquatiques recuillis en Espagne. Entomologiste 22 (6): 144-151.

BREHM, V., 1947. Reflexiones sobre relaciones zoogeográficas de la fauna de agua dulce de la Península Ibérica. P. Inst. Biol. Apl. N : 53-74.

CASADO, C., 1986 Composición y estructura de las comunidades de macroinvertebrados de un río intensamente regulado del Sistema Central: Río Lozoya (Cuenca del Tajo). Tesis Doctoral. Facultad de Ciencias, Univ. Autónoma de Madrid.

Cummins, K.W., 1972. What is a river? Zoological Description. In: River Ecology and Man: 33-52. Academic Press.

Da Terra, L.W., 1981. Lista faunística de tricópteros de Portugal. Biol. Soc. Port. ent. 12.

FitTKaU, E., 1986. Conocimiento actual sobre la colonización de la región tropical sudamericana por insectos acuáticos y su historia evolutiva, con especial referencia a los quironómidos. Ann. Mus. Nat. Valparaiso, 17: 97-103.

Garcla de Jalón, D., 1979. Estudio de las comunidades de macroinvertebrados del río Lozoya, especialmente del orden Trichoptera. Tesis Doctoral. Univ. Politécnica Madrid. 231 págs.

García de Jalón, D., 1980. Efecto del embalse de Pinilla (Madrid) sobre las comunidades de macroinvertebrados bentónicos del río Lozoya. Bol. Est. Central Ecol., 9 (17): 47-52. ICONA.

GarCía dE JALón, D., 1982a. Contribución a la zoosociología de los Tricópteros españoles. SHILAP 10: 75-78; 38: 157-165; 39: 237-244.

García de JaLón, D., 1982b. Los Trichoptera del río Lozoya. Bol. Asoc. Esp. Entom. 5: 41-58.

García de Jalón, D. \& M. González del Tánago, 1982. Introducción a una zoosociología del macrobentos en los nos de la Sierra de Guadarrama. Bol. Est. Central Ecol. 11 (21): 63-71.

Garcfa de Jalón, D. \& M. González del Tánago, 1986. Ephemeroptera, Plecoptera y Trichoptera de los principales ríos de Málaga. Actas II Simposio sobre el agua en Andalucía: 331-346.

González del Tánago, M., D. García de Jalón \& N. MarTÍNEZ ELCORO, 1979. Aplicación de algunos índices biológicos a diversos ríos españoles para la estimación de la calidad de sus aguas. Tec. Invest. Trat. 14 (1): 28-38.

González, M.A., D. Garcia de Jalón \& L.W. Da Terra, 1987. Faunistic studies on Iberian Trichoptera: A Historical Survey and Present State of Knowledge. Actas 5th Int. Symp. Trichoptera: $85-90$.

González Pena, G., M. Gonzalez Tánago \& D. Garcfa DE JALÓN, 1987. Los Simúlidos (Díptera) de los ríos Guadalhorce y Guadiaro (Málaga, SE España). Actas N Congreso Español Limnol.: 233-242. 
Haering, F.J., 1984. Estudio limnológico del embalse de Valmayor. Tesis Doctoral. Facultad de Ciencias, Univ. Complutense de Madrid.

HYNES, H.B.N., 1970. The ecology of running waters. Toronto Univ. Press. 555 págs.

LoBón, J., 1979. Biometría y crecimiento del barbo común en el embalse de El Atazar. Tesis de Licenciatura, Facultad de Ciencias. Univ. Autónoma Madrid.

Madrid Moreno, J., 1911. Datos para el estudio del plancton del río Lozoya. Bol. R. Soc. Esp. Hist. Nat. (B), V-II.

MALICKY, B., 1983. Chorological patterns and biome types of European Trichoptera and other freshwater insects. Arch. Hydrobiol. 96 (2): 223-244.

MARGalef, R., 1949. Datos para la hidrobiología de la Sierra de Guadarrama. P. Inst. Biol. Apl. 6: 5-21.

Margalef, R., 1955a. Comunidades bióticas de las aguas dulces del Noroeste de España, P. Inst. Biol. Apl. 21: 5-85.

MARGalef, R., 1955b. Contribución al estudio de la fauna de las aguas dulces del Noroeste de España. P. Inst. Biol. Apl. 21: $137-171$

Margalef, R., D. Planas, J. Armengol, A. Vidal, N. Prat, A. Guiset, J. Toja \& M. Estrada, 1976. Limnología de los embalses españoles. MOPU. Madrid. n. ${ }^{\circ} 123$.

Martínez de Pisón, E. \& J. López Martínez, 1986. Las Fluctuaciones Glaciares Pleistocenas en Guadarrama y Gredos. Libro guía de la excursión del Simposio sobre fluctuaciones climáticas durante el Cuaternario en las regiones del Mediterráneo Occidental. U.A.M.

Meléndez, B., 1958. Mapa geológico de España y Portugal. Paraninfo Ed. Madrid.

Minshall, G.W., 1988. Stream ecosystem theory: a global perspective. J.N. Am. Benthol. Soc. 7 (4): 263-288.

MiraClE, M.R., 1982. Biogeography of the zooplanktonic communities of Spain. Journal of Biogeography 9: 455-467.

Prat, N., M.A. Puig \& G. González, 1983. Predicció i control de la qualitat de les aigües dels rius Besos i Llobregat II. Estudis i monografies 9. Diputació de Barcelona.
Puig, M.A., G. González \& O. Soriano, 1984. Introducción al estudio de las comunidades macrobentónicas de los ríos asturianos: efemerópteros, plecópteros, tncópteros, simúlidos y quironómidos. Limnética 1: 187-196.

REISS, F., 1978. Sich abzeichrende Verbreitungsmuster in der palaarkfischen-palaotropischen Chironomidenfauna (Dipt.). Mitt. dt. Ges. allg. angew. Ent. 1: 72-76.

Resh, V.H., A.V. Brown, A.P. Covich, M.E. Gurtz, H.W. Li, G.W. Minshall, S.R. Reice, A.L. Sheldon, J.B. WALLACE \& R.C. WisSMAR, 1988. The role of disturbance in stream ecology. J. N. Am. Benthol. Soc. 7 (4): 433-455.

ReVEnGA, S., 1979. Introducción al estudio de las comunidades de zooplancton de los embalses de «El Vellón» y de «El Atazar». Tesis de Licenciatura. Facultad de Ciencias, Universidad Autónoma de Madrid.

Román, J., F. Troyano \& M. Santos Comendador, 1981. Aproximación a la evaluación de las cargas de $\mathrm{N}$ y $\mathrm{P}$ de los vertidos a los embalses de abastecimiento de distintas procedencias y de su grado de peligrosidad ante la eutrofización de aquella. Canal Isabel II

Schmid, F., 1952 Contribución a I'étude des Trichópteres d'Espagne. Pirinées 8: 627-295.

STANFORD, J.A. \& J.V. WARD, 1983. The serial discontinuity concept of lotic ecosystems. In: Dynamic of lotic ecosystems: 29-43.

URBISTONDO, R., 1980. La eutrofización de los embalses del Canal de Isabel II. Bol. Inform. Medio Amb. CIMA 15: 34-39.

Vannotte, R.L.; G.W. Minshall, K.W. Cummins, J.R. SEDELL \& C.E. CusHING, 1980. The river continuum concept. Can. Jour. Fish. Aquat. Science. 37 (1): 130-137.

WARD, J.V. \& J.A. STANFORD, 1983. The intermediate disturbance hypothesis. In: Dynamics of lotic ecosystems: 347 356. 